Revue de droit comparé du travail et de la sécurité sociale

$2 \mid 2020$

La vie personnelle du salarié

\title{
Tatouages sur les corps et corps à l'ouvrage : état de la jurisprudence au Québec
}

\section{Urwana Coiquaud}

\section{(2) OpenEdition}

1 Journals

Édition électronique

URL : https://journals.openedition.org/rdctss/1050

DOI : 10.4000/rdctss. 1050

ISSN : 2262-9815

Éditeur

Centre de droit comparé du travail et de la sécurité sociale

Édition imprimée

Date de publication : 1 juin 2020

Pagination : 15-23

ISSN : 2117-4350

\section{Référence électronique}

Urwana Coiquaud, «Tatouages sur les corps et corps à l'ouvrage : état de la jurisprudence au

Québec », Revue de droit comparé du travail et de la sécurité sociale [En ligne], 2 | 2020, mis en ligne le 01 novembre 2021, consulté le 11 novembre 2021. URL : http://journals.openedition.org/rdctss/1050 ;

DOI : https://doi.org/10.4000/rdctss. 1050

\section{cc)}

Revue de droit comparé du travail et de la sécurité sociale est mise à disposition selon les termes de la Licence Creative Commons Attribution - Pas d'Utilisation Commerciale - Pas de Modification 4.0 International. 


\title{
TATOUAGES SUR LES CORPS ET CORPS À L'OUVRAGE : ÉTAT DE LA JURISPRUDENCE AU QUÉBEC
}
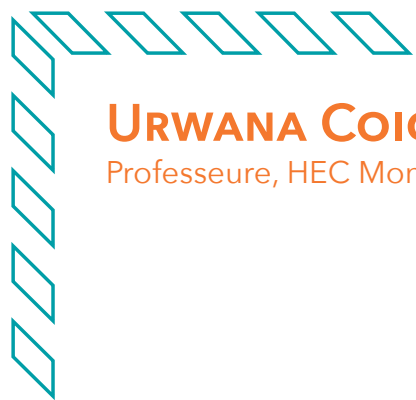

URWANA COIQUAUD

Professeure, HEC Montréal, membre du CRIMT

\section{RÉSUMÉ}

Le tatouage a franchi les frontières du monde interlope pour se hisser dans toutes les sphères de la société et permet à chacun d'exprimer son individualité. Si ce phénomène est devenu relativement banal et accepté dans nos sociétés et en particulier au Canada, qu'en est-il en contexte de travail? Quelles sont les garanties réglementaires accordées à l'employé tatoué? L'employeur peut-il imposer des règles entourant le port de tatouages? Le cas échéant, jusqu'où peut-il contraindre le salarié? Après avoir exposé le cadre réglementaire protecteur, de l'une de ses provinces, le Québec, nous examinerons le traitement jurisprudentiel qui est réservé à cette problématique.

Mots-clés : Liberté d'expression, vie privée, apparence physique, tatouage, droit du travail québécois

\begin{abstract}
Tattooing has crossed the borders of the underworld to reach into all spheres of society and allows everyone to express their individuality. If this phenomenon has become relatively commonplace and accepted in our societies, particularly in Canada, what about in the work context? What are the regulatory guarantees granted to the tattooed employee? Can the employer impose rules surrounding the wearing of tattoos? If so, to what extent can he or she compel the employee to do so? After outlining the protective regulatory framework of one of its provinces, Quebec, we will examine their treatment by the courts.
\end{abstract}

Key words: Freedom of Expression, a Right to Privacy, Physical Appearance, Tattoo, Quebec Employment Law 


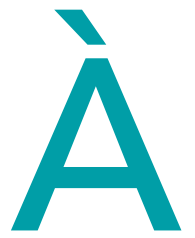

I'heure des médias sociaux, l'apparence physique n'a jamais eu autant d'importance qu'à ce jour. En témoigne le nombre infini de photos "léchées » et retouchées, publiées sans pudeur sur les réseaux sociaux. Dialogue constant entre la sphère de l'intime et du public, fruit d'un héritage biologique et social, l'apparence physique est un puissant véhicule de communication et de pouvoir.

Parmi ces véhicules, certains s'imposent à la vie, comme la couleur de peau ou la taille, car ils sont intrinsèques à l'individu'. D'autres sont pleinement contrôlés par l'individu comme la tenue vestimentaire, l'hygiène ou encore les bijoux, tandis que certains éléments le sont difficilement, car "[c]es caractéristiques sont difficiles à changer ou ne sont modifiables qu'à un prix personnel inacceptable $»^{2}$. Il peut s'agir du port d'un vêtement dont la signification religieuse est forte, comme le kirpan, ou encore du tatouage.

Le tatouage est réalisé au moyen d'un appareil : le dermographe. Composé de fines aiguilles, il martèle la peau en insérant de l'encre entre le derme et l'épiderme, marquant ainsi définitivement l'individu ${ }^{3}$. Longtemps perçu comme l'attribut des marginaux et désignant - encore souvent aujourd'hui - ceux qui veulent s'affranchir des interdits, le tatouage est surtout devenu l'expression d'un art, d'une mémoire, d'un pacte, de croyances, d'une histoire personnelle, d'une mode. Il a traversé les frontières du sexe en s'imposant chez les femmes, et il a franchi les frontières du monde interlope pour se hisser dans les plus hautes sphères sociales. Au final, le tatouage est devenu un art de vivre permettant d'exprimer son individualité ${ }^{4}$. Au Canada, environ 20\% de la population serait tatouée ${ }^{5}$.

Si ce phénomène est devenu relativement banal et accepté dans nos sociétés, qu'en est-il dans le contexte du travail? Quelles sont les garanties règlementaires accordées au salarié tatoué? L'employeur peut-il imposer des règles entourant le port de tatouages? Le cas échéant, jusqu'où peut-il contraindre le salarié?

1 A-M. Delagrave, Le contrôle de l'apparence physique du salarié, Cowansville, Québec, Éd. Y. Blais, 2010, p. 14.

2 Id., p. 16.

3 C. Rioult, "Le tatouage : un certain regard sur le corps », Journal français de psychiatrie, 2006, vol. 24, n¹, p. 44.

4 D. Le Breton, «Le monde à fleur de peau : sur le tatouage contemporain », Hermès, La Revue, 2016, vol. $74, n^{\circ} 1$, p. 132.

5 I. Morin, «La culture pop : planète tatouage », La Presse, 10 juillet 2015. 
Ce cadre est fermement délimité par la liberté d'expression et le respect de la vie privée garantis par la Constitution canadienne ${ }^{6}$ et la Charte des droits et libertés de la personne $e^{7}(\mathbf{I})$. Une analyse jurisprudentielle des arrêts de la Cour suprême du Canada et des décisions des niveaux inférieurs du Québec révèlera ensuite la mise en œuvre de ces normes et leurs interprétations par les tribunaux (II).

\section{I - LE SALARIÉ TATOUÉ : PROTECTION ET LIMITES RÈGLEMENTAIRES}

André Courchaine est chauffeur de bus syndiqué depuis 18 ans pour une société de transport. II porte un uniforme durant ses heures de travail. Le 5 janvier 2008, il s'est fait tatouer le côté droit de son visage, de façon très apparente. D'après lui, il s'agit là de son meilleur profil, mais aussi de celui qu'il présente à la clientèle lorsqu'elle monte à bord du bus. Selon son employeur, ce tatouage donne une image défavorable et contraire à ce que la société cherche à projeter auprès de sa clientèle. L'employeur suggère donc que ce tatouage soit retiré et, en attendant une solution idoine, prend la décision de muter le chauffeur. Ce dernier estime, quant à lui, qu'aucune directive n'existait à ce propos et qu'il s'agit d'un choix personnel. À ses yeux, ce geste ne concerne pas l'employeur et l'ôter laisserait des marques cutanées pires que le tatouage lui-même.

Ce cas $^{8}$ illustre clairement le conflit possible entre l'expression des droits et libertés fondamentaux d'un salarié tatoué et les droits de l'employeur. La suite de cette analyse se concentrera sur la protection des droits et libertés fondamentaux du salarié tatoué, reconnus par la Charte des droits et libertés de la personne (A) et les atteintes possibles de l'employeur (B).

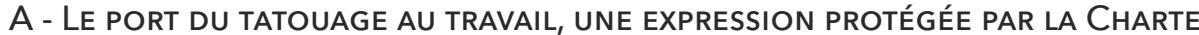 DES DROITS ET LIBERTÉS DE LA PERSONNE}

Le contrôle de l'apparence physique exercé par l'employeur porte atteinte aux droits et libertés fondamentaux des travailleurs. Deux droits sont particulièrement visés, le droit à la vie privée et à la liberté d'expression : "Le droit à la liberté d'expression repose sur la conviction que la libre circulation des idées et des images est la meilleure voie vers la vérité, l'épanouissement personnel et la coexistence pacifique dans une société hétérogène composée de personnes dont les croyances divergent et s'opposent. Si nous n'aimons pas une idée ou une image, nous sommes libres de nous y opposer ou simplement de nous

6 Charte canadienne des droits et libertés, Loi de 1982 sur le Canada, Annexe B, 1982 (R.-U.), ch. 11 (ci-après citée : «Charte canadienne »). Le régime fédératif institué par la Constitution canadienne répartit la compétence législative entre le Parlement du Canada et les législatures des provinces ou des territoires. Les lois sur les droits de la personne ont été promulguées aux deux paliers (fédéral et provinciaux). Au Québec, il s'agit de la Charte des droits et libertés de la personne (RLRQ, c. C-12) (ci-après citée: " Charte québécoise »), un texte de valeur quasi constitutionnelle.

7 Ce texte, contrairement à la Charte canadienne, s'applique aux relations de nature privée.

8 Syndicat des chauffeurs de la S.T.L. et Société de Transport de Laval, DTE 2009T-92 [2009] RJDT 290 [ci-après «STL »]. 


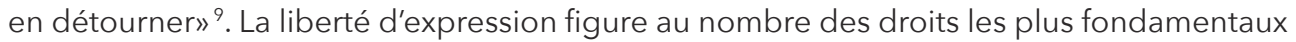
des Canadiens et le tatouage, en raison de sa portée expressive, est visé « par l'article 3 de la Charte québécoise $»^{10}$. Tel est ce qui est affirmé par les tribunaux spécialisés. Le tatouage est considéré comme " un piège à regard » ${ }^{11}$, qui exprime une certaine ambivalence - celle de « montrer tout en cachant, mais aussi de cacher tout en montrant $»^{12}$ - et constitue un moyen d'envoyer un message à l'autre, " de lancer une bouteille à la mer que l'écoute et le regard analytique permettent de déchiffrer $»^{13}$.

Outre la liberté d'expression, le tatouage est aussi protégé par le droit au respect de la vie privée. L'article 5 de la Charte québécoise protège le droit de prendre des décisions fondamentalement personnelles, sans pression externe indue. Le tatouage fait donc partie de cette sphère d'autonomie individuelle ${ }^{14}$, car " à n'en pas douter, le choix d'une personne [...] de porter sur son corps une marque indélébile fait partie de ces décisions relevant de la sphère d'autonomie protégée par le droit à la vie privée $»^{15}$. Dans l'affaire, Fraternité des policiers et policières de Saint-Jean-sur-Richelieu et St-Jeansur-Richelieu (Ville de $)^{16}$, le juge conclut : «les tribunaux ont reconnu que le droit à l'image est une composante du droit à la vie privée (...) [et qu'il] fait partie du droit à l'image. II s'insère dans cette sphère d'autonomie individuelle relativement à l'ensemble des décisions qui se rapportent à des « choix de nature fondamentalement privée ou intrinsèquement personnelle » [réf. omise].

Ainsi, lorsque l'employeur décide de contrôler l'apparence physique du salarié via l'adoption d'un règlement interne, d'une disposition contractuelle, d'une directive ou encore d'une conduite, il contraint l'exercice de ses droits et libertés fondamentaux ${ }^{17}$. La démonstration par le salarié d'une atteinte prima facie à une liberté ou un droit fondamental imposera à l'employeur de la justifier.

\section{B - LA JUSTIFICATION DE L'ATTEINTE AUX LIBERTÉS ET DROITS FONDAMENTAUX DU SALARIÉ}

Une fois la preuve apportée par le salarié de la violation d'un droit ou d'une liberté, il revient à l'employeur de démontrer que celle-ci est justifiée en vertu de l'article 9.1 de la Charte québécoise. À cet égard, il est important de rappeler que l'exercice du droit de

9 Ford c. Québec (Procureur général), [1988] 2 R.C.S. 712, §. 21 [ci-après « Ford »].

10 Parent c. 9000-5489 Québec inc., DTE 97T-665 (CQ), p. 6 [ci-après « Parent »].

11 C. Rioult, ibid., p. 44.

12 ld.

13 ld.

14 Godbout c. Longueuil (Ville), [1997] 3 R.C.S. 844, p. 913; Aubry c. Éditions Vice-Versa inc., [1998] 1 R.C.S. 591, p. 614.

15 Syndicat des travailleuses des centres de la petite enfance du Saguenay-Lac-St-Jean - FSSS-CSN c. Girard, C.S., 2009-05-27, § 28 (Requête en révision judiciaire accueillie, appel rejeté 2011 QCCA 1620), [ci-après « Girard »].

162016 QCTA 715 [ci-après «St-Jean-sur-Richelieu »].

17 C. Brunelle et M. Samson, "Les droits et libertés dans le civil », Droit public et administratif, Collection droit, 2019-2020, Vol. 8, 2019, Cowansville, éd. Yvon Blais, p. 4. 
direction de l'employeur ${ }^{18}$ n'est pas absolu et reste assujetti à la primauté de la Charte, dont la valeur est quasi constitutionnelle:

9.1. Les libertés et droits fondamentaux s'exercent dans le respect des valeurs démocratiques, de la laïcité de l'État, de l'ordre public et du bien-être général des citoyens du Québec. La loi peut, à cet égard, en fixer la portée et en aménager l'exercice.

La Cour suprême du Canada ${ }^{19}$, dans les affaires R. c. Oakes ${ }^{20}$ et RJR-MacDonald Inc. c. Canada (Procureur général)21, a établi deux critères :

1) Lorsqu'il y a atteinte à un droit ou une liberté fondamentaux, il faut en premier lieu se demander si l'exigence ou la restriction formulée par l'employeur à l'égard de l'apparence physique du salarié répond à un objectif légitime et important. Cet objectif doit être suffisamment important car les juges précisent que la norme doit être sévère ${ }^{22}$ et qu'il faut « à tout le moins qu'un objectif se rapporte à des préoccupations urgentes et réelles dans une société libre et démocratique, pour qu'on puisse le qualifier de suffisamment important ${ }^{23}$. À cet égard, il a été admis que «le tatouage, d'une façon générale, dans la société actuelle (...) ne constitue pas une valeur morale ou une culture si discutable qu'il peut être restreint pour le respect des valeurs démocratiques, pour l'ordre public et le bien-être des citoyens ${ }^{24}$.

2) Deuxièmement, la partie qui réclame cette restriction doit démontrer que la mesure contestée a un lien rationnel avec l'objectif poursuivi, c'est-à-dire que « les moyens choisis sont raisonnables et que leur justification peut se démontrer. Cela nécessite l'application du critère de proportionnalité. Les tribunaux devront, dans chaque cas, soupeser les intérêts en présence. Ce critère de proportionnalité doit être évalué selon les critères suivants : «même à supposer qu'il y ait un tel lien rationnel, le moyen choisi doit être de nature à porter "le moins possible" atteinte au droit ou à la liberté en question (...) il doit y avoir proportionnalité entre les effets des mesures restreignant un droit ou une liberté garantis par la Charte et l'objectif reconnu comme "suffisamment important" „25.

C'est à la lumière de ces principes, que les tribunaux rendent leurs décisions.

18 La question du fondement juridique du pouvoir de l'employeur fait l'objet d'importants débats qui dépassent ici le cadre de cette analyse jurisprudentielle.

19 Ces critères ont été élaborés dans l'arrêt R. c. Oakes, [1986] 1 RCS 103 [ci-après " Oakes »], en regard de l'article 1 de la Charte canadienne et, dans la mesure où la disposition justificative de l'article 9.1 al. 2 de la Charte québécoise s'apparente à cet article, il est admis que ce test s'applique aussi à la Charte québécoise : Ford c. Québec (Procureur général), [1988] 2 R.C.S. 712, p. 769.

20 Ibid., Oakes, note 19.

21 [1995] 3 RCS 199 [ci-après «RJR »].

22 Ibid., Oakes, § 69.

$23 \mathrm{ld}$.

24 Ibid., Parent, p. 12.

25 Ibid., Oakes, note 19, § 70. 


\section{I - LE TATOUAGE DU SALARIÉ À L'ÉPREUVE DES EXIGENCES DE L'EMPLOYEUR}

Les juges doivent dans le cadre des affaires soumises vérifier si les objectifs poursuivis par l'employeur sont légitimes (A) et si les solutions proposées sont raisonnables et justifiées (B).

\section{A - Les ObJeCtifs LÉGitimes de L'EMPLOYEuR}

Dans le cadre de rapports individuels ou collectifs de travail, la jurisprudence admet qu'en vertu de son droit de direction, l'employeur peut émettre des restrictions - convention collective, code de conduite, directive orale ou écrite - concernant l'apparence personnelle des salariés. Ces restrictions doivent poursuivre des objectifs légitimes et importants, à savoir "une raison d'affaire légitime $»^{26}$ ou une "expression commerciale $»^{27}$. En outre, l'employeur peut justifier de telles atteintes pour protéger la santé et de la sécurité des salariés, celle du public ou celle de l'entreprise ${ }^{28}$, même si le test paraît plus rigoureux lorsque la justification repose sur cette dernière justification ${ }^{29}$. Or, c'est précisément sur ce dernier élément que s'appuient les employeurs pour restreindre le port du tatouage qui heurte, dans la quasi-totalité des cas, l'image de l'entreprise.

Ainsi, un Centre de jeunesse ${ }^{30}$, garantissant la protection de jeunes dont la sécurité ou le développement est compromis, peut restreindre certains attributs de l'apparence personnelle des salariés, afin de projeter une image professionnelle cohérente avec les valeurs et la mission de l'établissement: " s'assurer que la clientèle vulnérable ne se retrouve pas dans une position où les enseignements de l'institution seraient contredits par l'image véhiculée par ses propres employés ॥' $^{31}$.

De la même façon, un Centre de la petite enfance (CPE), qui fournit des services éducatifs aux enfants de 0 à 5 ans, peut être amené à contraindre l'apparence physique des éducateurs et éducatrices de manière à protéger les enfants contre des signes sexistes, racistes, incitant à la violence ou faisant la promotion de l'alcool ou de la drogue ${ }^{32}$. Par exemple, la mission d'un tel organisme pourrait être heurtée par une éducatrice arborant sur l'avant-bras un tatouage représentant un doigt d'honneur devant une bouche entrouverte.

26 Commission des Droits de la Personne, Les exigences des employeurs et des établissements de service sur la tenue vestimentaire et l'apparence personnelle, M. Drapeau, (Cat. 2.113- 3.6), 1993, p. 8 : http://www.cdpdj.qc.ca/Publications/uniforme.pdf

27 Ibid., Ford, note 9, § 50 : selon cet arrêt, "L'expression commerciale " mérite une protection constitutionnelle « en raison du rôle important qu'elle joue en facilitant les choix économiques éclairés ».

28 ld., p. 9.

29 Id., p. 10.

30 Syndicat canadien de la Fonction publique, section locale 4268 et Centre jeunesse de Montréal institut universitaire, 2012 CanLII 99872 (OC SAT) [ci-après « Centre jeunesse de Montréal »].

31 ld., p. 33.

32 Ibid., Girard, note 15, § 33. 
Le caractère offensant et connoté sexuellement dudit tatouage pourrait contrevenir à la mission éducative du $\mathrm{CPE}^{33}$.

Un service de police souhaite protéger son image afin de maintenir la confiance et le respect des citoyens envers lui, conformément au Code de déontologie. Il faut « éviter que les policiers aient l'air marginaux, ou briser le lien de confiance de la population envers les policiers par la présence d'un tatouage qui serait trop visible, d'où la restriction associée à la taille des tatouages, à leur nombre et à leur emplacement ${ }^{34}$. Cet objectif s'avère suffisamment important pour restreindre la visibilité des tatouages du policier ${ }^{35}$.

Un service public de transport qui s'adresse à une population de tous âges et de toutes les cultures peut imposer des normes d'apparence physique en raison du contact quotidien des chauffeurs avec la clientèle, car l'image de l'entreprise passe beaucoup par l'impression que donnent les chauffeurs ${ }^{36}$.

Cette étape 1 du test franchie, l'employeur doit démontrer à l'étape 2 que la contrainte qu'il impose a un lien rationnel avec l'objectif poursuivi et que la limitation au droit, ou à la liberté, est minimale (c'est-à-dire que les moyens choisis sont raisonnables) et proportionnelle à l'objectif.

Dans le cadre d'un service de police, l'employeur devra démontrer que le tatouage va à l'encontre des obligations et devoirs du policier visant à « maintenir la paix, l'ordre, la sécurité publique, prévenir et réprimer le crime $\aleph^{37}$. Ainsi interdire un tatouage à connotation blasphématoire est une limitation rationnelle puisque le Code de déontologie prévoit que le policier ne peut faire usage de langage obscène, blasphématoire ou injurieux. De la même façon, l'interdiction d'arborer un tatouage comportant une image ou un symbole associé, ou faisant la promotion de la criminalité, comporte lui aussi un lien rationnel.

Mais quelle qualité de preuve doit-il fournir? S'agira-t-il d'une preuve scientifique comme un sondage d'opinion rigoureux? Si l'arrêt Oakes avait parlé de preuve «forte et persuasive » (paragraphe 68), la Cour suprême a, depuis lors, assoupli ce critère ${ }^{38}$. La preuve doit être objective et ne peut reposer sur une simple appréhension ou croyance de

33 Lupien et Centre de la petite enfance Grande-Rivière, 2019 QCTAT 610 (CanLII) : le recours entrepris n'a pas permis au tribunal de déterminer l'atteinte aux droits et libertés de la salariée.

34 Saint-Jean-sur-Richelieu, Ibid., note 16, § 81-82.

35 Fraternité des policiers et policières de Longueuil et Ville de Longueuil, EYB2019-3049049, § 64 [ci-après « Longueuil »].

36 Ibid., STL, note 8, § 116.

37 Ibid., Longueuil, note 35, § 68.

38 Ibid., RJR, § 138 : «Pour satisfaire à la norme de preuve en matière civile, on n'a pas à faire une démonstration scientifique; la prépondérance des probabilités s'établit par application du bon sens à ce qui est connu, même si ce qui est connu peut comporter des lacunes du point de vue scientifique ». 
l'employeur. Elle doit néanmoins être précise et rigoureuse ${ }^{39}$, et prendre en considération l'évolution sociétale ${ }^{40}$. II ne s'agit pas d'une preuve scientifique, mais logique et rationnelle (paragraphe 72$)^{41}$.

Il convient aussi d'examiner la portée de l'interdiction formulée par l'employeur. Par exemple, si l'interdiction porte sur tous les tatouages sans discernement, l'employeur échoue à cette étape. Ainsi, un Centre de la petite enfance ${ }^{42}$ qui accueille des enfants n'est pas justifié à licencier tout salarié tatoué, sans égards à l'endroit ou à la nature du tatouage.

Pour que l'atteinte soit minimale, il est important qu'en présence de politiques d'entreprise, les salariés comprennent ce qui est permis, ou ne l'est pas. Par exemple, un juge a estimé qu'une politique d'entreprise interdisant un tatouage comportant une "caractéristique qui va à l'encontre de l'une des valeurs du service de police, à savoir le service à la population, le professionnalisme, le respect, l'intégrité et la oyauté " ou à " connotation violente " sont des termes trop vagues ${ }^{43}$ et offrent trop de discrétion à l'employeur ${ }^{44}$. De ce fait, les tribunaux rappellent l'importance d'émettre des politiques d'entreprise claires, conformes au cadre règlementaire, connues de tous, dont l'application reste constante et les conséquences identiques en cas de non-respect ${ }^{45}$, à défaut de quoi l'employeur ne pourra que sanctionner ${ }^{46}$.

À la rigueur des tests imposés, le tribunal ajoute qu'il lui revient de soupeser les effets négatifs de la mesure par rapport aux avantages liés à l'objectif de l'employeur.

\section{B - RECOUVRIR, FAIRE DISPARAITRE UN TATOUAGE, OU CHANGER D'AFFECTATION, UNE EXIGENCE RAISONNABLE?}

Si dissimuler tout tatouage, quel qu'en soit le contenu et la localisation sur le corps, constitue une atteinte injustifiée, car disproportionnée par rapport à l'objectif sérieux et important de restreindre la liberté ou le droit fondamental du salariée ${ }^{47}$, qu'en est-il de la

39 Ne répond pas à ces critères un sondage concernant « les perceptions et attitudes de la clientèle des supermarchés à l'égard du body piercing »: affaire Travailleuses et travailleurs unis de l'alimentation et du commerce, section locale 486 et Provigo Distribution inc. (Division Maxi \& Cie Gatineau), 2001, CanLII 59310 (QC SAT). Postérieurement à cette affaire, un juge note en obiter dictum qu'un sondage effectué selon les règles « aurait donné une indication très sérieuse sur la décision à prendre en l'espèce, mais il serait très dangereux de s'y fier à 100\%. Ce serait lui donner un pouvoir énorme qui pourrait aboutir à des injustices importantes », STL, note 8, § 116 (ibid.).

40 Ibid., Saint-Jean-sur-Richelieu, note 16, § 90. Bien qu'à cet égard, il faille nuancer selon l'endroit, l'ampleur et la nature expressive du tatouage; par exemple, une tête de mort tatouée sur le visage n'aura pas le même impact qu'une étoile encrée sur la face interne de la cheville.

41 lbid., Longueuil, note $35, \S 72$.

42 Ibid., Parent, note 13, p. 13.

43 Ibid., Longueuil, note $35, \S 86-87$.

44 ld.

45 Re Lumber \& Sawmill Workers' Union, Local 2537 and KVP Co. Ltd., (1965) 16 L.A.C. 73 (J.B. Robinson).

46 Ibid., STL, note 8, § 123.

47 Ibid., Girard, note 15. 
politique qui exige de couvrir certains tatouages en raison de leur localisation sur le corps ou du message qu'ils véhiculent?

Dans l'affaire Centre jeunesse de Montréal ${ }^{48}$, la politique de l'employeur tolère les tatouages en précisant toutefois que "lorsqu'ils se situent dans certaines zones du corps (c'est à dire sur le haut d'un sein ou au haut de l'arc fessier), ils ne doivent pas être exhibés ou, autrement dit, on doit les couvrir» ${ }^{49}$. Ici, l'employeur exige la dissimulation du tatouage qui apparaît à certains endroits dits intimes ou à connotation sexuelle. Cette restriction se fonde essentiellement sur des motifs de décence et d'exemplarité à l'égard des jeunes, afin d'éviter d'attirer l'attention tant sur le tatouage que sur la partie du corps concernée. Le juge estime que l'objectif est sérieux et important, la restriction rationnellement liée à l'objectif et minimale puisque seules certaines parties du corps sont visées. Finalement, l'inconvénient de dissimuler n'est pas disproportionné par rapport à l'importance de l'objectif.

Dans une autre affaire, une policière arborait un nouveau tatouage. Situé sur l'ensemble de la face externe de la main droite allant du poignet aux jointures des doigts, il représente une tête de mort dont les orbites et la cavité nasale sont prédominantes. "Cette image portée de façon visible sur la main devient celle du service de police, et l'employeur est en droit de vouloir préserver son image ${ }^{50}$. Le visage et la main sont les premiers contacts du policier avec le citoyen. Parce que la tête de mort est associée à la criminalité et à la violence, ce tatouage contrevient à la directive de l'employeur et peut affecter la confiance, la considération et le respect des citoyens. L'employeur exige donc de le couvrir avec un gant de cycliste lorsqu'elle est en contact avec les citoyens, un inconvénient majeur à ses yeux, car il instaure un climat de méfiance pour certains citoyens tandis que, pour la population marginalisée, la vue de son tatouage crée au contraire une proximité qui facilite le contact. Dès lors, le juge estime que la mesure imposée (port du gant) n'est pas proportionnée. II reviendra donc aux parties de trouver une solution pour couvrir le tatouage sans les inconvénients relatés, car ils aboutissent tous deux à une perte de confiance de la population envers le corps policier.

Outre le camouflage, d'autres mesures d'accommodement sont-elles envisageables? Dans l'affaire $S T L^{51}$, le chauffeur a tatoué son visage et son geste quasi irréversible a conduit l'employeur à proposer deux solutions : celle d'enlever le tatouage, ou d'assigner le chauffeur à d'autres fonctions. En l'espèce, le juge n'envisage aucune des deux solutions dans la mesure où la première présente trop d'inconvénients et la deuxième ne peut s'imposer vu l'absence de diligence de l'entreprise. En effet, la mesure d'accommodement ne peut le priver de son travail de chauffeur, alors même que l'entreprise aurait dû imposer une politique en amont.

\section{Conclusion}

Aujourd'hui le tatouage est devenu un trait de l'apparence physique des plus communs. II s'invite sur la plage, dans les bars, mais aussi au travail. Dans un tel contexte, les analyses règlementaire et jurisprudentielle révèlent les droits et libertés fondamentaux du salarié

48 Ibid., note 30.

49 ld., $\S 188$.

50 ld., $\$ 116$.

51 Ibid., STL, note 8, § 123. 


\section{QUÉBEC}

susceptibles d'être atteints, et le test rigoureux auquel doit se soumettre l'employeur lorsqu'il décide d'y porter atteinte. À l'issue de cette étude, trois constats peuvent être dégagés.

La jurisprudence a accompagné l'évolution sociétale et révèle à ce titre l'exercice " prudent d'un pouvoir créatif [celui des juges], considéré comme légitime et nécessaire pour l'évolution de la société ${ }^{52}$.

En dépit de ce premier constat positif, il faut relever le silence souvent gênant des décideurs autour du fondement juridique du droit de l'employeur de contrôler l'apparence physique des salariés, et ce même si plusieurs travaux doctrinaux s'y attaquent.

Finalement, l'entièreté des cas de jurisprudence analysés dans cette étude concerne des travailleurs protégés par des conventions collectives, preuve qu'en contexte non syndiqué, il peut être périlleux de faire valoir ses droits. Et que dire des candidats tatoués éconduits et de la persistance des stéréotypes et des stigmas attestée par la littérature ${ }^{53}$ ? La jurisprudence reste aussi silencieuse à leur propos, signe que le droit doit encore apprivoiser et combattre ces silences.

52 L. Lebel, L'art de juger, B. Melkevik (dir.), Collection Dikè, Québec, PUL, 2019, p. 24.

53 A. R. Timming, "Visible Tattoos in the Service Sector: A New Challenge to Recruitment and Selection », 2015, vol. 29, n¹, Work, Employment and Society, p. 60. 